\title{
p53 expression in dyskeratosis congenita: a marker for oral premalignancy?
}

\author{
G R Ogden, D P Lane, D M Chisholm
}

\begin{abstract}
As p53 expression has been associated with malignant disease its presence was assessed in biopsy specimens from dorsal lingual hyperkeratosis, taken over a five year period. p53 expression, using CM1, was assesssed using a standard immunoperoxidase technique. p53 was not identified in the first biopsy specimen in 1986 but was identified in all subsequent ones. Only in the latest biopsy specimen was there evidence for dysplasia in haematoxylin and eosin stained sections.

It is suggested that p53 expression may be a reliable marker for predicting premalignant change in keratoses occurring in dyskeratosis congenita.
\end{abstract}

(F Clin Pathol 1993;46:169-170)

The syndrome dyskeratosis congenita consists of a triad of leucoplakia of the mucous membranes, increased skin pigmentation, and nail dystrophy. The variation in the manifestations of this syndrome are discussed elsewhere. ${ }^{1}$ The syndrome is rare and usually proves fatal, either as a result of gross haematological disturbance or malignant change within the keratoses. ${ }^{1}$ To date, there is no reliable method for predicting which lesions will undergo malignant change.

We have previously reported ultrastructural observation, ${ }^{2}$ and an analysis of the keratin profile in biopsy specimens taken over a five year period, ${ }^{3}$ in an attempt to identify a marker for potential malignant change.

Much attention has recently focused on the identification of p53 in a variety of malignant tumours, ${ }^{4}$ and its absence from normal or benign lesions. ${ }^{5}$ The accumulation of p53 seems to be a common step in the development of most human cancers. ${ }^{4}$ However, identification of p53 using immunocytochemistry may occasionally occur in non-malignant tissue. For example, we have identified p 53 positive cells in tissue sections taken from some odontogenic keratocysts. ${ }^{7}$ However, when using $\mathrm{CM} 1$, we have never detected p53 in benign oral mucosal conditions. ${ }^{8}$

Given that with regard to oral mucosal lesions p53 has only been detected in oral carcinomas, it was proposed that such expression be assessed in sequential biopsy specimens taken from an oral keratosis occurring in dyskeratosis congenita, for evidence of potential malignant change.

\section{Methods}

The biopsy specimens were obtained from a boy aged 10 in 1986, who was diagnosed as having dyskertosis congenita: principally, oral keratoses, nail dystropy, and increased skin pigmentation (as previously reported ${ }^{1}$ ). Biopsy specimens from the dorsal tongue lesion taken in $1986,1987,1989$ and 1991 were available and haematoxylin and eosin stained sections were taken from each. The sections were then cut from formalin fixed, paraffin wax embedded tissue, dewaxed, and washed in TRISbuffered saline (TBS). The sections were blocked with goat serum (diluted 1 in 5) for 30 minutes before incubation overnight at $4^{\circ} \mathrm{C}$ with the polyclonal antibody CM1 (diluted 1 in 1000 of $10 \%$ fetal calf serum in phosphate buffered saline). CM1 is a rabbit polyclonal antibody raised against the whole $\mathrm{p} 53$ protein expressed in Escherichia coli. ${ }^{9}$ Sections were washed in running tap water. A biotinylated anti-rabbit immunoglobulin was applied for one hour before applying the avidin:biotin complex (Vectastain, Vector Labs, Peterborough, England) for one hour at room temperature. The specimens were then incubated with a solution of diaminobenzidine tetrahydrochloride (DAB) and hydrogen peroxide in TBS for 10 minutes. The presence of p53 was identified by a brown precipitate within the nucleus. Negative controls comprised the use of CM1 absorbed with $\mathrm{p} 53$ and the substitution of normal goat serum in place of CM1.

\section{Results}

No positive staining occurred in the 1986 biopsy specimen incubated with CM1. However, in all subsequent biopsy specimens p53 was positively identified in the basal cells (figs 1 and 2).

In all biopsy specimens except that taken in 1991 no evidence for dysplasia at light microscopic level was seen. Mild basal cell hyperplasia, a patchy chronic inflammatory cell infiltrate, and orthokeratosis were the main features (fig 1B). However, the biopsy specimen taken in 1991 revealed moderate to severe epithelial dysplasia, with some evidence for candidal infection.

\section{Discussion}

p53 expression in oral mucosal lesions has previously only been identified in malignant disease. ${ }^{8}$ Its identification in the biopsy specimens taken in 1987 and 1989, in which no obvious histopathological evidence for dysplasia was noted, is therefore intriguing. Not least because a later biopsy specimen (taken in 1991) was diagnosed as a carcinoma in situ.

We have previously found evidence for dis-
Accepted for publication 7 August 1992 
Figure $1 A$ Expression of p53 in the basal cells of the dorsal lingual

hyperkeratotic lesion taken in 1987, using CM1.

Figure $1 B$ Section of the biopsy specimen taken in 1987 (haematoxylin and eosin.)

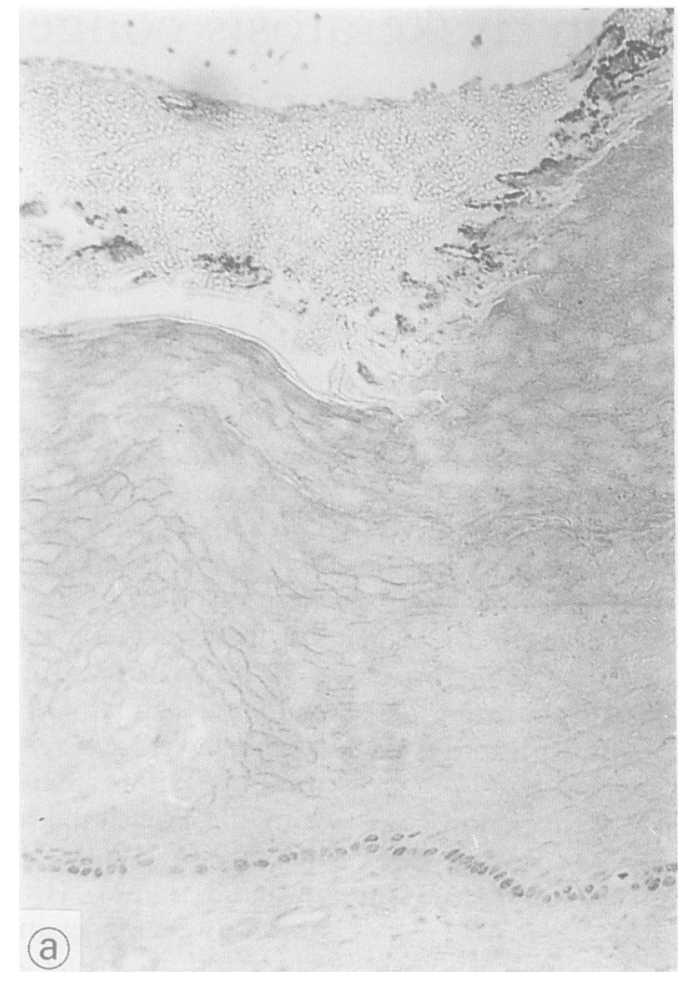

turbed epithelial maturation in this case of dyskertosis congenita, both at ultrastructural examination $^{2}$ and by cytoskeletal morphometry. ${ }^{3}$

Fine structural analysis of the dorsal lingual hyperkeratosis of this case, whose clinical features have previously been reported $^{1}$ revealed an apparent increase in nucleolar number as the only clinically relevant finding. $^{2}$

Analysis of the keratin cytoskeleton revealed more important changes ${ }^{3}$; principally, the coexpression of three type 1 keratins (K16, $\mathrm{K} 10$, and $\mathrm{K} 13$ ) in the lingual keratosis, and the

Figure 2 CM1 staining of the basal and parabasal cells in the dorsal hyperkeratotic lesion taken in 1991

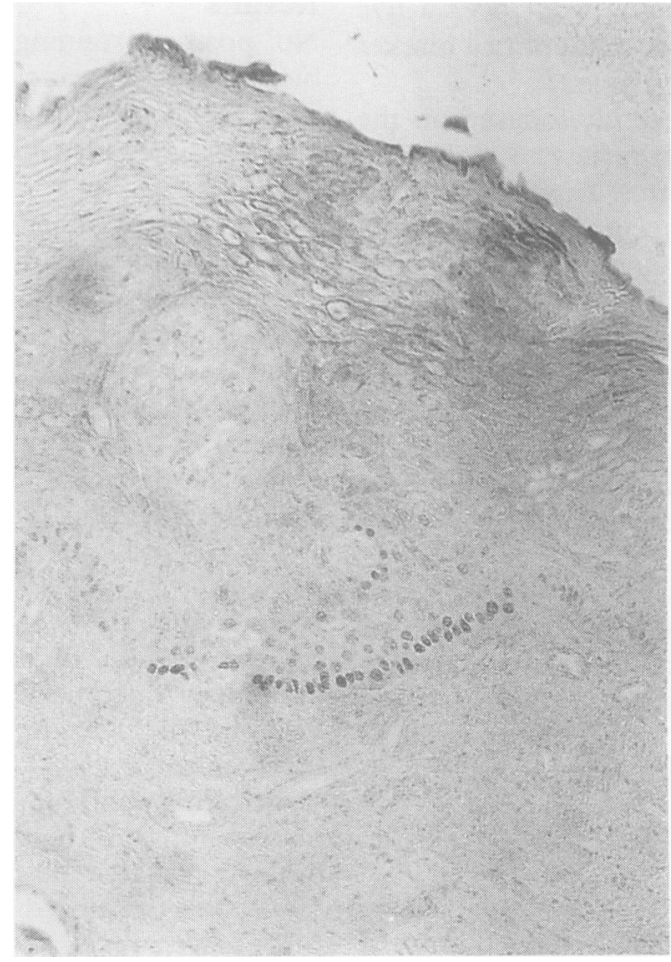

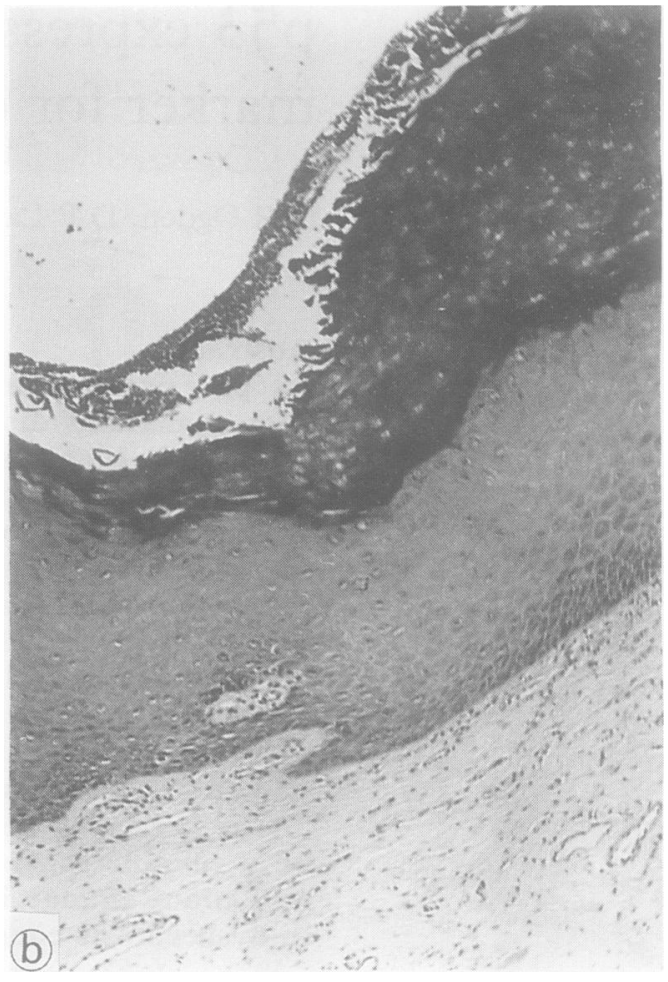

identification of $\mathrm{K} 8$ (using CAM5.2) in the basal cells of the dorsal tongue keratosis. Keratin 8 is usually only found in nonkeratinocytes in tissue sections of normal oral mucosa, although such expression has been noted in some oral squamous carcinomas. ${ }^{10}$ These keratin profiles were suggestive of an unusually immature or disturbed state of tissue differentiation.

Because the immunohistochemical identification of p53 is usually associated with malignant disease, its identification four years before any histopathological evidence for dysplasia suggests that it may be a useful marker for identifying those lesions which have a propensity to undergo malignant change. These findings may have implications for the clinical management of patients with oral manifestations of dyskeratosis congenita.

1 Ogden GR, Connor E, Chisholm DM. Dyskeratosis congenita; a report of a case and review of the literature. Oral Surg Oral Med Oral Pathol 1988;65:586-91.

2 McKay GS, Ogden GR, Chisholm DM. Lingual hyperkeratosis in dyskeratosis congenita-preliminary ultrastructural report. F Oral Pathol Med 1991;20:196-9.

3 Ogden GR, Chisholm DM, Leight IM, Lane EB. Cytokeratin profiles in dyskeratosis congenita: an immunocytochemical investigation of lingual hyperkeratosis. $₹$ Oral Pathol Med 1992;21:353-7.

4 Lane DP. Mutation of the p 53 gene and accumulation of the p53 protein: Common steps found in the majority of human cancers (1991). In: Fortner J G, Rhoads JC, eds. General Motors Cancer Research Foundation accomplishments in cancer research 1990. Philadelphia: J B Lippincott

5 Hall PA, Ray A, Lemoine NR, Midgley CA, Krausz T, Lane DP. p53 immunostaining as a marker of malignant disease in diagnostic cytopathology. Lancet 1991;338:513.

6 Wynford-Thomas D. p53 in tumour pathology: can we trus immunocytochemistry? ₹ Pathol 1992;166:329-30.

7 Ogden GR, Chisholm DM, Kiddie RA, Lane DP. p53 protein in odontogenic cysts: increased expression in some odontogenic keratocysts. F Clin Pathol 1992;45: $1007-10$.

8 Ogden GR, Kiddie RA, Lunny DP, Lane DP. Assessment of p53 protein expression in normal, benign, and malignan oral mucos. 7 Pathol 1992:166:389-94.

9 Midgley CA, Fisher CJ, Bartek J, Vojtesek B, Lane DP, Barnes DM. Analysis of p53 expression in human tumours. An antibody raised against human p53 expressed in E coli. $¥$ Cell $S c i$ 1992;101:183-9.

10 Morgan PR, Shirlaw PJ, Johnson NW, Leigh IM, Lane EB. Potential applications of antikeratin antibodies in oral diagnosis. $\mathcal{f}$ Oral Pathol 1987;16:212-22. 